\title{
Dietary fat and physiological determinants of plasma chylomicron remnant homoeostasis in normolipidaemic subjects: insight into atherogenic risk
}

\author{
Deasy Irawati ${ }^{1,2,3}$, John C. L. Mamo ${ }^{1,2}$, Karin M. Slivkoff-Clark ${ }^{1}$, Mario J. Soares ${ }^{1,2}$ and \\ Anthony P. James ${ }^{1,2 *}$ \\ ${ }^{1}$ School of Public Health, Faculty of Health Sciences, Curtin University, Perth, WA 6102, Australia \\ ${ }^{2}$ Curtin Health Innovation Research Institute, Faculty of Health Sciences, Curtin University, Perth, WA 6102, Australia \\ ${ }^{3}$ Faculty of Medicine, Mataram University, West Nusa Tenggara 83125, Indonesia
}

(Submitted 1 September 2016 - Final revision received 20 December 2016 - Accepted 15 January 2017 - First published online 20 February 2017)

\section{Abstract}

TAG depleted remnants of postprandial chylomicrons are a risk factor for atherosclerosis. Recent studies have demonstrated that in the fasted state, the majority of chylomicrons are small enough for transcytosis to arterial subendothelial space and accelerate atherogenesis. However, the size distribution of chylomicrons in the absorptive state is unclear. This study explored in normolipidaemic subjects the postprandial distribution of the chylomicron marker, apoB-48, in a TAG-rich lipoprotein plasma fraction (Svedberg flotation rate (Sf $>400$ ), in partially hydrolysed remnants (Sf 20-400) and in a TAG-deplete fraction ( $\mathrm{Sf}<20)$, following ingestion of isoenergetic meals with either palm oil (PO), rice bran or coconut oil. Results from this study show that the majority of fasting chylomicrons are within the potentially pro-atherogenic $\mathrm{Sf}<20$ fraction $(70-75 \%)$. Following the ingestion of test meals, chylomicronaemia was also principally distributed within the $\mathrm{Sf}<20$ fraction. However, approximately $40 \%$ of subjects demonstrated exaggerated postprandial lipaemia specifically in response to the SFA-rich PO meal, with a transient shift to more buoyant chylomicron fractions. The latter demonstrates that heterogeneity in the magnitude and duration of hyper-remnantaemia is dependent on both the nature of the meal fatty acids ingested and possible metabolic determinants that influence chylomicron metabolism. The study findings reiterate that fasting plasma TAG is a poor indicator of atherogenic chylomicron remnant homoeostasis and emphasises the merits of considering specifically, chylomicron remnant abundance and kinetics in the context of atherogenic risk. Few studies address the latter, despite the majority of life being spent in the postprandial and absorptive state.

\section{Key words: Dietary fatty acids: Chylomicron remnants: ApoB-48: Postprandial lipaemia}

The majority of humans spend most of their 24-h-d in the postprandial state. A typical $24 \mathrm{~h}$ TAG profile in individuals consuming three to four meals and snacks per $\mathrm{d}$, showed a $70 \%$ increase in TAG concentration that reached baseline for only $3 \mathrm{~h}$ in the early morning ${ }^{(1)}$. Therefore, humans are essentially in a constant state of absorptive lipaemia. Following ingestion of dietary fats there is an elevation of TAG-enriched chylomicrons, however this elevation is ordinarily transient. Insulinstimulated expression of lipoprotein lipase (LPL) bound to glycosylphosphatidylinositol anchored HDL-binding protein 1 mediates the hydrolysis of TAG from chylomicrons, resulting in rapid release of NEFA that can be taken up by endothelial cells via the membrane protein cluster of differentiation $36^{(2)}$. The post-hydrolysed TAG depleted remnants of chylomicrons and VLDL, are then removed via high-affinity receptor pathways, principally in the liver ${ }^{(3)}$.

Postprandial hypertriacylglycerolaemia is indicative of the net effect of dietary lipid absorption, biogenesis and secretion of de novo chylomicrons and lipolysis by endothelial lipases. Modest intakes of dietary TAG can be mostly accommodated in the constitutively secreted chylomicrons, and may result in only minor changes to particle number ${ }^{(4,5)}$. However, rather than the TAG-rich lipoproteins (TRL) secreted following fat ingestion, accumulation of the post-hydrolysed remnants in plasma poses a greater atherogenic risk ${ }^{(6)}$. Indeed, fasting hypertriacylglycerolaemia, or postprandial dyslipidaemia, is not a requisite surrogate marker of chylomicron remnant accumulation per $s e^{(7-9)}$, and measurement of chylomicron particle number, rather than TAG concentration provides a more complete marker of pro-atherogenic remnant accumulation $^{(10)}$

Regulating the plasma abundance of pro-atherogenic chylomicron remnants principally relies on lipoprotein clearance capacity through receptor mediated pathways ${ }^{(11)}$. Chylomicrons share the lipolytic cascade and high affinity clearance pathways with TRL of hepatic origin (VLDL). An accumulation of VLDL

Abbreviations: CO, coconut oil; HR, hyper-responder; IAUC, incremental AUC; LPL, lipoprotein lipase; NR, normo-responder; PO, palm oil; RBO, rice bran oil; Sf, Svedberg flotation rate

* Corresponding author: Dr A. P. James, fax +61 89266 2958, email T.P.James@curtin.edu.au 
remnants, or indeed LDL as a consequence of receptor insufficiency, often serves as a surrogate marker of clearance deficits of chylomicron remnant lipoproteins, because chylomicron remnants are primarily removed from blood by the apo $\mathrm{B} / \mathrm{E}$ receptor (LDL-receptor). Although apo B-48, the equivocal marker of chylomicron abundance is not always reported, it is reported to be substantially elevated in subjects with diabetes/ the metabolic syndrome, familial hypertriacylglycerolaemia, familial hypercholesterolaemia and in normolipidaemic subjects with coronary artery disease ${ }^{(12-15)}$. Lipoproteins that are reduced to remnants with a diameter of $<70 \mathrm{~nm}$ contribute to atherosclerosis through their capacity to deliver cholesterol to the subendothelial space and retention in extracellular matrices ${ }^{(16-18)}$. Indeed, fully hydrolysed chylomicron remnants with diameters of $55 \mathrm{~nm}$ have been reported and contain approximately forty times more cholesterol than LDL per particle ${ }^{(19,20)}$.

In healthy Caucasians, the plasma distribution of fasting apo B-48 is principally realised in the small, dense and potentially more atherogenic Svedberg flotation $(\mathrm{Sf})<20$ lipoprotein fraction $^{(21)}$. However, in this study, we report that this abundance was not normally distributed, suggesting inter-individual variability in basal rates of chylomicron synthesis and/or remnant clearance. Moreover, there is likely to be potential interactive effects with dietary fats and the genetic regulation of chylomicron homoeostasis $^{(22)}$. However, the hypothesis of differential synergistic fatty acid/genetically mediated effects on chylomicron remnant abundance per se has never been explored directly.

Dietary fatty acid type has been reported to influence chylomicron metabolism by modulating chylomicron assembly (i.e. microsomal transfer protein activity, lipid droplet formation and stability), LPL mediated hydrolysis and high-affinity clearance pathways ${ }^{(23,24)}$. Among the different types dietary fatty acid there is an abundance of evidence that SFA are pro-atherogenic via pathways that include undefined aberrations in chylomicron metabolism; a broader hypercholesterolaemic induction effect and subsequently heightened vascular inflammation ${ }^{(25-28)}$. Differential effects of fatty acid type on postprandial lipaemia have been widely reported. Exaggerated postprandial lipaemia may be due to overproduction of intestinally derived and hepatically derived TRL, slower clearance by LPL, a rateregulatory hydrolysis and removal of TAG or accumulation of partially hydrolysed remnant lipoproteins ${ }^{(1,23,24)}$. However, the comparative response to different dietary fats on chylomicron size and importantly remnant abundance is less clear. To address the latter, this study was designed to explore plasma apo B-48 distribution in normolipidaemic but otherwise healthy subjects given different meals rich in either SFA, long-chain $n-6$ PUFA or in medium chain fatty acids.

\section{Methods}

This crossover designed study was conducted at Curtin University, Australia, between August 2014 and December 2015 and approved by the Curtin University Human Research Ethics Committee (HR 151/2013). All participants provided signed informed consent at initial screening. The study was registered with Australian New Zealand Clinical Trials Registry (12614000352606).

\section{Subjects}

Male and female healthy participants aged between 20 and 70 years were recruited by means of standard media and web portals. Before acceptance in the study, potential interested participants were invited to meet the investigators and attended an initial screening. The exclusion criteria were fasting TAG $1.7 \mathrm{mmol} / \mathrm{l}$ or more, pregnant, lactating, smoking, excess alcohol intake $(>20 \mathrm{~g}$ alcohol/d), the use of lipid lowering medication, reported history of cardiovascular, hepatic, renal disease, gastric disturbances or use of drug that affect gastrointestinal motility, diabetes mellitus, unwillingness to avoid consumption of fish oil supplements $3 \mathrm{~d}$ before and during the study days, $> \pm 5 \mathrm{~kg}$ changes in body weight in the previous 6 months, on a dietary advice and allergy or intolerance to any ingredients of the test meals.

Subjects were randomised using the random-number generator function RANDBETWEEN in Microsoft Excel software for the order of the test meals. Sample size required was determined from the sample size of twenty-four in a comparable study in which difference in apo B-48 postprandial response between postprandial hyperlipidaemia and normolipidaemia subjects were detected with a power of $80 \%$ and $\alpha$ of $0.05^{(29)}$.

\section{Test meal composition}

Each participant consumed an isoenergetic breakfast meal $(3100 \mathrm{~kJ}, 43.4 \mathrm{~g}$ fat, $69.4 \mathrm{~g}$ carbohydrate and $13.6 \mathrm{~g}$ protein) on three occasions in which $40 \mathrm{~g}$ of the fat was from one of the following three different cooking oils: palm oil (PO) (Bimoli; PT Indofood Sukses Makmur Tbk), coconut oil (CO) (Eco Food Organics) and rice bran oil (RBO) (Alfa One ${ }^{\mathrm{TM}}$ Rice Bran Oil; Hansell Food Group) (Table 1). The test meal was a mixed breakfast meal consisting of pasta (150 g) (Large spiral pasta; San Remo Pasta Ltd), tomato pasta sauce (50 g) (Dolmio Traditional Recipe Classic Tomato Pasta Sauce; Mars Food) added with $40 \mathrm{~g}$ test oils, white bread ( $40 \mathrm{~g}$ ) (white toaster; Mias Bakery) and $160 \mathrm{ml}$ orange juice (Just Juice, orange juice). Each meal provided $53 \%$ of total energy as fat. Fatty acid content of the different oils is provided in Table 1 . Fatty acid composition of the cooking oils were analysed in the form of fatty acid

Table 1. Fat content of the test oils measured by GC

\begin{tabular}{|c|c|c|c|c|c|c|c|c|c|}
\hline $\begin{array}{l}\text { Fatty acid } \\
\text { content (\%) }\end{array}$ & $\begin{array}{l}\text { Total } \\
\text { SFA }\end{array}$ & $\begin{array}{l}\text { Total } \\
\text { MUFA }\end{array}$ & $\begin{array}{l}\text { Total } \\
\text { PUFA }\end{array}$ & $\begin{array}{c}12: 0 \\
\text { (lauric acid) }\end{array}$ & $\begin{array}{c}14: 0 \\
\text { (myristic acid) }\end{array}$ & $\begin{array}{c}16: 0 \\
\text { (palmitic acid) }\end{array}$ & $\begin{array}{c}18: 0 \\
\text { (stearic acid) }\end{array}$ & $\begin{array}{c}18: 1 n-9 \\
\text { (oleic acid) }\end{array}$ & $\begin{array}{c}18: 2 n-6 \\
\text { (linoleic acid) }\end{array}$ \\
\hline Palm oil & 60 & 31 & 9 & 0.1 & $4 \cdot 3$ & $42 \cdot 1$ & $9 \cdot 8$ & $30 \cdot 4$ & 8.8 \\
\hline Coconut oil & 92 & 8 & 0 & $47 \cdot 2$ & $27 \cdot 2$ & $13 \cdot 3$ & 4.4 & $7 \cdot 8$ & 0.0 \\
\hline Rice bran oil & 28 & 40 & 32 & 0.1 & 0.1 & 24.7 & $3 \cdot 2$ & 39.9 & $32 \cdot 2$ \\
\hline
\end{tabular}


methyl esters by GC (Perkin Elmer AutoSystem XL) equipped with an autosampler, a split-splitless injector, SGE BPX70 GC column and a flame ionisation detector using a method developed in our laboratory.

\section{Study protocol}

Participants attended three postprandial study days separated by at least 4 weeks between each visit. Before each study day, participants were asked to restrain from vigorous physical activity, alcohol and consumption of high-fat meals. They were asked to consume the same amount and food items for breakfast and lunch on the day before each postprandial test day and were provided with a standard commercially available ready meal (SunRice pack meal, $15 \mathrm{~g}$ fat; SunRice) for their evening meal before study day. After an overnight fast for at least $12 \mathrm{~h}$, participants were admitted to the research unit. Upon arrival, their body weight, height, waist circumference and blood pressure (Omron) were measured. An initial fasting blood samples were collected before consumption of the test meal. Following consumption of the test meal, participants were given access to plain water with no other food and drinks to be consumed. The test meal was consumed within $15 \mathrm{~min}$ and subsequently postprandial blood samples were collected periodically at 4 and 8-h after the test meal for measurement of apo B-48, TAG and cholesterol concentration. Body composition measured by bioelectrical impedance analysis (SECA mBCA 515; SECA GmbH \& Co. KG), was determined on the first fat challenge. Fasting and postprandial venous blood samples were collected into BD Vacutainer $^{\mathrm{R}}$ EDTA tubes (Becton Dickinson) and underwent low-speed centrifugation. Plasma samples were isolated and stored in aliquots at $-80^{\circ} \mathrm{C}$.

\section{Lipoprotein fractionation}

Sequential floatation ultracentrifugation was used to collect lipoprotein fractions of Sf $>400$ and Sf $20-400$ of plasma samples using a modified method that has been used in our laboratory ${ }^{(30,31)}$. In brief, $1 \mathrm{ml}$ plasma was overlayed with a density solution $(1.006 \mathrm{~g} / \mathrm{ml})$ in $5 \mathrm{ml}$ thin walled tubes (Beckman Coulter). The buoyant chylomicron fraction ( $\mathrm{Sf}>400)$ was collected following $30 \mathrm{~min}$ of ultracentrifugation at $40000 \mathrm{rpm}, 20^{\circ} \mathrm{C}$ (AH-650 swingout rotor; Thermo Scientific) by aspirating approximately $1 \mathrm{ml}$ of the top layer. To collect Sf 20-400, the tube was then topped with density solution $(1.006 \mathrm{~g} / \mathrm{ml})$ and ultracentrifuged again under the same condition for $20.5 \mathrm{~h}$. Approximately $1 \mathrm{ml}$ of the top layer was aspirated to isolate this fraction. The infranatant remaining in the tube was regarded as the remnant fraction $(\mathrm{Sf}<20)$. To calculate the concentrations of apo B-48, TAG and cholesterol of these fractions, the volume of all supernatants and infranatant collected were taken into account by measuring the collected supernatants or infranatant using a glass bore syringe (Hamilton).

\section{Lipid, insulin and glucose assays}

Fasting and postprandial TAG and total cholesterol in plasma and lipoprotein fractions were measured with an enzyme-based colorimetric reagent (Trace; Thermo Fisher Scientific Inc.; CV $<5 \%$ ).
HDL was collected using a precipitation method by the addition of phosphotungstic acid in the presence of Mg ions (HDL-cholesterol precipitant Reagent; Randox Laboratories Limited). A modified version of Friedewald formula was used to estimate LDLcholesterol $^{(32)}$. Fasting insulin and glucose concentrations were analysed by an accredited clinical laboratory (PathWest Laboratory, Fiona Stanley Network) using their automated procedures. The level of insulin resistance was assessed by homoeostasis model of insulin resistance (HOMA-IR) ${ }^{(33)}$. All samples were assayed in duplicate and run within a single batch.

\section{Apo B-48 determination}

Apo B-48 concentration was measured by using a commercial sandwich ELISA method (Shibayagi Human apo B-48 ELISA Kit; Ishihara; $\mathrm{CV}<3 \%$ ). This method has been validated ${ }^{(34)}$. The determination of apo B-48 concentration in plasma, Sf $>400$ and Sf 20-400 fractions was completed based on the manufacturer's instruction with some modification on the dilution factors to ensure that the apo B-48 concentration in those fractions within the standard curve. Fasting and postprandial plasma, Sf $>400$ and Sf 20-400 samples were 1 in 300, 1 in 50 and 1 in 100 diluted, respectively. Apo B-48 concentration in $\mathrm{Sf}<20$ was calculated from the difference between the concentration of apo B- 48 in plasma and in the less dense fractions (Sf > 400 and Sf 20-400).

\section{Statistical analysis}

All data were analysed using SPSS version 21 (SPSS Inc.). Data were checked for normality and were natural log transformed where necessary. Postprandial response (incremental AUC (IAUC)) was calculated using the trapezoidal rule ${ }^{(35)}$ with some modifications. The IAUC was estimated as the difference between the area under the plasma curve between 0 and $8 \mathrm{~h}$ and the area defined below the baseline concentration. However in responses where the $8 \mathrm{~h}$ concentration was lower than that at baseline, the baseline AUC represented the area below a line connecting the baseline and $8 \mathrm{~h}$ concentration levels. The IAUC represents the increase in area after the response of the fat load above fasting concentrations. The primary outcome of this study was IAUC apo B-48 in plasma, Sf $>400$, Sf 20-400 and Sf $<20$ fraction. The secondary outcome of this study was IAUC TAG in plasma and lipoprotein fractions and the association between IAUC apo B-48 and a number of metabolic determinants. Postprandial differences between groups following each meal challenge were assessed with Mann-Whitney $U$ test. Differences in postprandial responses between meals (PO, $\mathrm{CO}$ and $\mathrm{RBO}$ ) were analysed by linear mixed model with Bonferroni post hoc tests to detect significant pairwise differences corrected for multiple comparisons. Spearman correlations were computed to assess the association between parameters. A $P$ value $<0.05$ was considered as significant.

\section{Results}

Totally, twenty-six participants with normal fasting TAG concentration took part in the single blind, randomised crossover 
study, of which twenty-three completed all three meal challenges. In all, three subjects only attended two postprandial visits. None of the participants reported any adverse effects to the test meals. To explore putative interactive effects of the dietary fat challenge with a genetically determined chylomicron distributional response, subjects were considered based on the postprandial TAG response $4 \mathrm{~h}$ after consuming the positive lipaemic control SFA-enriched meal (PO) were examined. Subjects were classified as hyper-responders (HR) if plasma TAG exceeded $1.7 \mathrm{mmol} / \mathrm{l}$ at the peak absorptive phase of $4 \mathrm{~h}$. Of the twenty-six subjects studied, ten had concentrations $\geq 1.7 \mathrm{mmol} / \mathrm{l}$ (HR). Totally, sixteen subjects were found to have a 4 -h TAG concentration $<1.7 \mathrm{mmol} / \mathrm{l}$ following the SFA-enriched meal (normo-responders (NR))

Table 2 shows that the HR and NR groups had comparable fasting apo B-48. In addition, HR and NR groups were normolipidaemic and comparable for plasma TAG, total cholesterol, LDL-cholesterol and non-HDL-cholesterol. The HR group had a lower concentration of HDL-cholesterol than NR, however this was otherwise within the normal reference range. Both NR and HR groups were normoglycaemic and there was no evidence of insulin resistance based on HOMA or fasting plasma insulin. However, the HR group had significantly greater BMI, waist circumference and fat-free mass than the NR group.

The putative effect of dietary fatty acids on the distribution of chylomicrons in lipoprotein fractions in NR and HR subjects is depicted in Fig. 1. In NR, the plasma IAUC for apo B-48 was comparable for the PO-enriched fat challenge $v$, the RBO diet (23.9 (SE 4.9) $\mu \mathrm{g} \times 8 \mathrm{~h} / \mathrm{ml}$ in PO $v .27 .2$ (SE 3.9) $\mu \mathrm{g} \times 8 \mathrm{~h} / \mathrm{ml}$ in RBO) (meal effect $P=0 \cdot 068$ ). However, there was a modestly lower plasma apo B-48 IAUC response in NR subjects following consumption of the CO diet (18.4 (SE 2.6) $\mu \mathrm{g} \times 8 \mathrm{~h} / \mathrm{ml}$ ). In contrast, HR subjects had approximately 2-3-fold greater IAUC apo B-48 plasma response following ingestion of PO (63.2 (sE 13.4) $\mu \mathrm{g} \times 8 \mathrm{~h} / \mathrm{ml}$ ) relative to the meal challenge with $\mathrm{RBO}$ $(35.3(\mathrm{sE} \quad 6.2) \mu \mathrm{g} \times 8 \mathrm{~h} / \mathrm{ml})$ or $\mathrm{CO}(24.4 \quad(\mathrm{sE} 4.8) \mu \mathrm{g} \times 8 \mathrm{~h} / \mathrm{ml})$ (meal effect $P=0.003$, PO-CO $P=0 \cdot 016$, PO-RBO $P=0 \cdot 267$ ).

Distributional analysis of the heightened apo B-48 IAUC in HR subjects following ingestion of the PO-enriched meal, indicated that approximately half of the exaggerated apo B-48 response was realised in the remnant $\mathrm{Sf}<20$ fraction $(32 \cdot 1$ (SE 6.6$) \mu \mathrm{g} \times 8 \mathrm{~h} / \mathrm{ml}$ in HR $v .13 .4$ (SE 4.3$) \mu \mathrm{g} \times 8 \mathrm{~h} / \mathrm{ml}$ in $\mathrm{NR}$; $P=0.02$ ). Smaller increases in the apo B-48 IAUC were also observed in HR subjects compared with NR within the Sf 20-400 fraction $(14.4(\mathrm{SE} 4.5) \mu \mathrm{g} \times 8 \mathrm{~h} / \mathrm{ml}$ in HR $v .6 .6$ (sE 1.4$) \mu \mathrm{g} \times 8 \mathrm{~h} / \mathrm{ml}$ in NR; $P=0.138$ ) and the larger more buoyant lipoprotein isolate $\mathrm{Sf}>400(17.3(\mathrm{SE} 4.8) \mu \mathrm{g} \times 8 \mathrm{~h} / \mathrm{ml}$ in HR $v .4 \cdot 2$ (SE 1.4$) \mu \mathrm{g} \times 8 \mathrm{~h} / \mathrm{ml}$ in NR, respectively; $P=0.024$ ). Distributional analysis of the apo B-48 IAUC in HR and NR subjects following ingestion of a meal rich in either $\mathrm{RBO}$ or $\mathrm{CO}$, did not indicate significant differences between lipoprotein fractions. This finding is consistent with the notion that the disturbed response was specific to the PO fat challenge in HR subjects.

The TAG response in lipoprotein fractions for NR and HR subjects in response to the dietary fat challenges is indicated in Fig. 2. In NR subjects, the plasma IAUC TAG response following the PO meal $(1.7(\mathrm{SE} 0.3) \mathrm{mmol} \times 8 \mathrm{~h} / \mathrm{l})$ was comparable with the response following ingestion of $\mathrm{RBO}(2 \cdot 1$ (SE $0 \cdot 6) \mathrm{mmol} \times 8 \mathrm{~h} / \mathrm{l})$

Table 2. Subject characteristics

(Mean values with their standard errors; medians, 25th and 75th interquartile (IQR) ranges)

\begin{tabular}{|c|c|c|c|c|c|c|}
\hline \multirow[b]{2}{*}{ Parameters } & \multicolumn{2}{|c|}{ Total $(n$ 26) } & \multicolumn{2}{|c|}{ NR $(n 16)$} & \multicolumn{2}{|c|}{$\mathrm{HR}(n 10)$} \\
\hline & Mean & SE & Mean & SE & Mean & SE \\
\hline Men/women & \multicolumn{2}{|c|}{$11 / 15$} & \multicolumn{2}{|c|}{$4 / 12$} & \multicolumn{2}{|c|}{$7 / 3$} \\
\hline Age (years) & 41 & 3 & 39 & 4 & 43 & 6 \\
\hline \multicolumn{7}{|l|}{ Aро B-48 $(\mu \mathrm{g} / \mathrm{ml})$} \\
\hline Median & \multirow{2}{*}{\multicolumn{2}{|c|}{$\begin{array}{c}6 \cdot 3 \\
4.8-9.4\end{array}$}} & \multirow{2}{*}{\multicolumn{2}{|c|}{$\begin{array}{c}7 \cdot 1 \\
5 \cdot 2-11 \cdot 3\end{array}$}} & \multirow{2}{*}{\multicolumn{2}{|c|}{$5 \cdot 1$}} \\
\hline 25th and 75th IQR & & & & & & \\
\hline TAG $(\mathrm{mmol} / \mathrm{l})$ & 1 & 0.1 & 0.9 & 0.1 & $1 \cdot 1$ & 0.1 \\
\hline Total cholesterol $(\mathrm{mmol} / \mathrm{l})$ & 5.5 & 0.2 & 5.4 & 0.3 & 5.4 & 0.4 \\
\hline LDL-cholesterol (mmol/l) & 3.3 & 0.2 & 3.2 & 0.3 & 3.6 & 0.4 \\
\hline HDL-cholesterol (mmol/l) & $1 \cdot 7$ & 0.1 & 1.8 & 0.1 & 1.4 & $0.1^{*}$ \\
\hline Non-HDL-cholesterol (mmol//) & 3.8 & 0.2 & 3.6 & 0.3 & $4 \cdot 1$ & 0.4 \\
\hline Glucose $(\mathrm{mmol} / \mathrm{l})$ & 5.5 & 0.1 & 5.5 & 0.1 & 5.5 & $0 \cdot 1$ \\
\hline \multicolumn{7}{|l|}{ Insulin (mIU/l) } \\
\hline Median & \multirow{2}{*}{\multicolumn{2}{|c|}{$\begin{array}{c}6 \cdot 8 \\
4 \cdot 2-11 \cdot 1\end{array}$}} & \multicolumn{2}{|c|}{6.8} & \multicolumn{2}{|c|}{7} \\
\hline 25th and 75th IQR & & & & & & \\
\hline \multicolumn{7}{|l|}{ HOMA-IR } \\
\hline Median & \multicolumn{2}{|c|}{$1 \cdot 7$} & \multicolumn{2}{|c|}{$1 \cdot 7$} & \multicolumn{2}{|c|}{1.7} \\
\hline 25th and 75th IQR & \multicolumn{2}{|c|}{$1-3$} & \multicolumn{2}{|c|}{$1-2 \cdot 3$} & \multicolumn{2}{|l|}{$1 \cdot 3-3 \cdot 2$} \\
\hline Waist circumference $(\mathrm{cm})$ & 83.7 & $2 \cdot 7$ & $77 \cdot 9$ & 3.2 & 93.1 & $2 \cdot 8^{\star \star}$ \\
\hline $\mathrm{BMI}\left(\mathrm{kg} / \mathrm{m}^{2}\right)$ & $25 \cdot 8$ & 0.8 & $24 \cdot 1$ & 1 & $28 \cdot 6$ & $0.9^{\star \star}$ \\
\hline Fat mass index $\left(\mathrm{kg} / \mathrm{m}^{2}\right)$ & 7.7 & 0.7 & $7 \cdot 2$ & 1 & 8.5 & 1.1 \\
\hline Fat-free mass index $\left(\mathrm{kg} / \mathrm{m}^{2}\right)$ & 18 & 0.5 & $16 \cdot 8$ & 0.5 & 19.9 & $0.7^{\star \star}$ \\
\hline Systolic blood pressure (mmHg) & 122 & 3 & 119 & 4 & 127 & 5 \\
\hline \multicolumn{7}{|l|}{ Diastolic blood pressure (mmHg) } \\
\hline Median & \multirow{2}{*}{\multicolumn{2}{|c|}{$\begin{array}{c}72 \\
67-78\end{array}$}} & \multirow{2}{*}{\multicolumn{2}{|c|}{$\begin{array}{c}71 \\
67-77\end{array}$}} & \multirow{2}{*}{\multicolumn{2}{|c|}{$\begin{array}{c}73 \\
68-81\end{array}$}} \\
\hline 25th and 75th IQR & & & & & & \\
\hline
\end{tabular}

NR, normo-responder; HR, hyper-responder; HOMA-IR, homoeostasis model of insulin resistance.

${ }^{*} P<0.05,{ }^{*} P<0.01$; HR compared with NR group. $P$ values were based on independent $t$ test and Mann-Whitney test for mean and medians. 
(a)

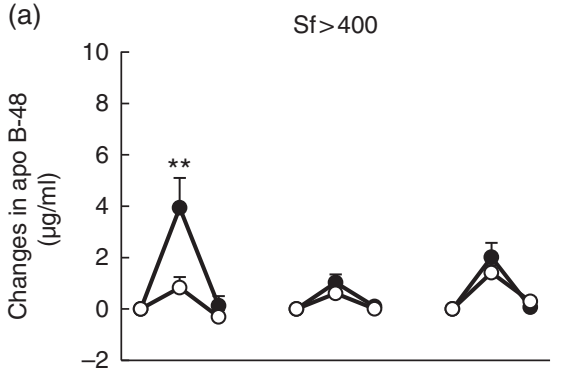

(c)

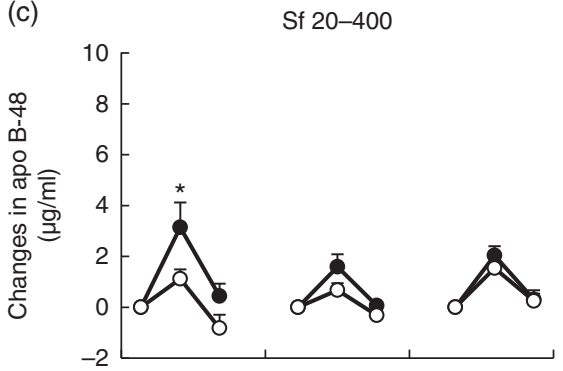

(e)

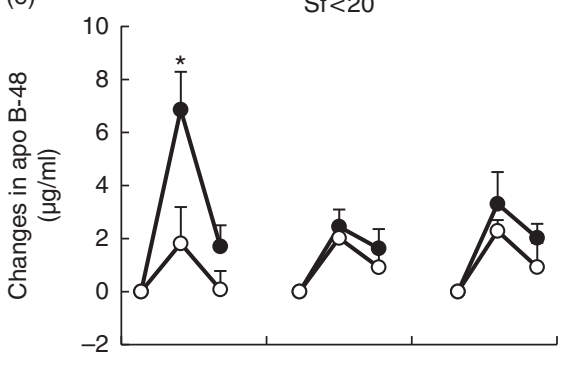

(g)

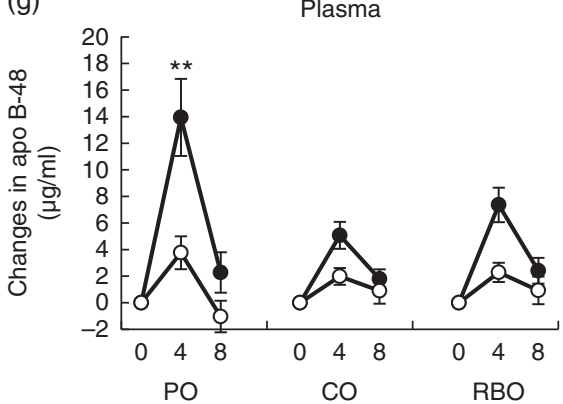

(b)

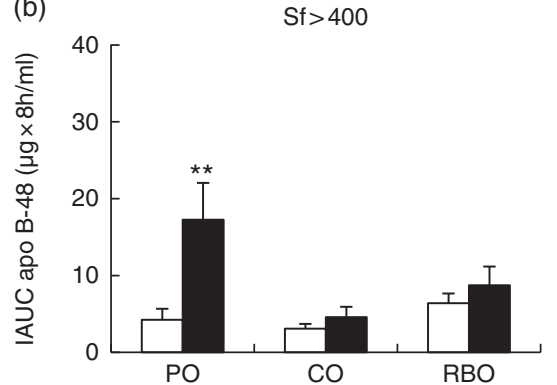

(d)

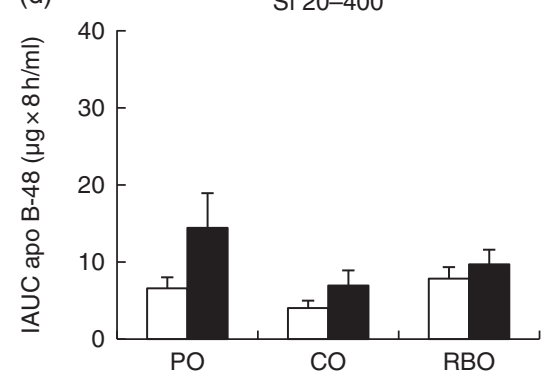

(f) $\mathrm{Sf}<20$

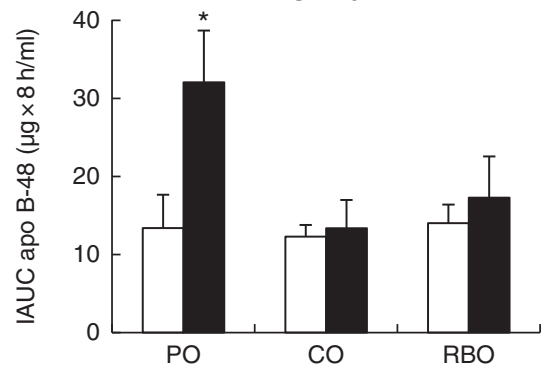

(h)

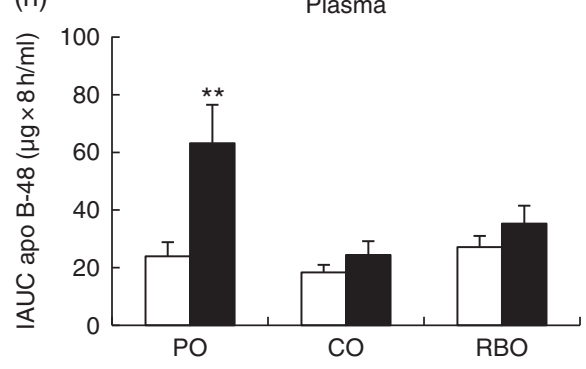

Fig. 1. Changes in apo B-48 concentration ((a), (c), (e), (g)) and incremental AUC (IAUC) of apo B-48 ((b), (d), (f), (h)) in Svedberg flotation rate (Sf) $>400$, Sf 20-400, $\mathrm{Sf}<20$ fraction and plasma following mixed meal containing palm oil (PO), coconut oil (CO) and rice bran oil (RBO). $\rightarrow$ and represent hyper-responder (HR) group; $\mathrm{O}$ and $\square$ represent normo-responder (NR) group. ${ }^{*} P$ value $<0.05,{ }^{* *} P$ value $<0.01$, against NR group.

or $\mathrm{CO}(1.4(\mathrm{se} 0.3) \mathrm{mmol} \times 8 \mathrm{~h} / \mathrm{l})$ (meal effect $P=0.414)$. In contrast, in HR subjects the IAUC response for TAG following the PO-enriched meal was substantially greater than either the $\operatorname{RBO}(4 \cdot 3(\operatorname{se~} 0 \cdot 7) v \cdot 2 \cdot 8(\operatorname{se~} 0.5) \mathrm{mmol} \times 8 \mathrm{~h} / \mathrm{l}$, respectively) or the CO-enriched meal $(1.7$ (sE 0.5$) \mathrm{mmol} \times 8 \mathrm{~h} / \mathrm{l}$ ) (meal effect $P=0.001$ ). Distributional analysis of the heightened TAG IAUC response indicated in HR subjects was principally realised with a 3 -fold increase in the largest and most buoyant lipoprotein fraction Sf $>400$ compared with $\mathrm{NR} \quad(3.1 \quad(\mathrm{SE} \quad 0 \cdot 6) \quad v .0 .9$ $(\mathrm{SE} 0 \cdot 2) \mathrm{mmol} \times 8 \mathrm{~h} / \mathrm{l}$, respectively; $P<0 \cdot 0005)$. Some increase in
TAG IAUC was also observed within the Sf 20-400 fraction of HR subjects compared with $\mathrm{NR}\left(\begin{array}{lllll}1.3 & \text { (sE } & 0.3\end{array}\right) \quad v .0 .5$ (sE $0 \cdot 1) \mathrm{mmol} \times 8 \mathrm{~h} / \mathrm{l}$, respectively; $P=0 \cdot 003$ ). Although relatively modest in the context of total TAG response, within the $\mathrm{Sf}<20$ fraction there was less of a TAG response in HR subjects compared with NR subjects $(0 \cdot 1$ (SE 0.2) v. 0.4 (SE 0.2) mmol $\times$ 8 h/l, respectively; $P=0 \cdot 272$ ).

Suggested metabolic determinants of chylomicron homoeostasis in HR and NR subjects is depicted in Table 3. Fat-free mass determined by bioelectrical impedance analysis was 
(a)

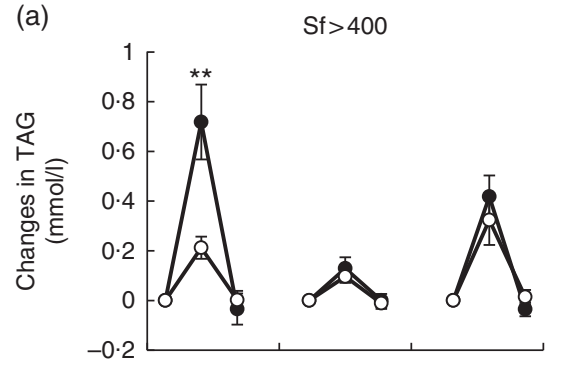

(c)

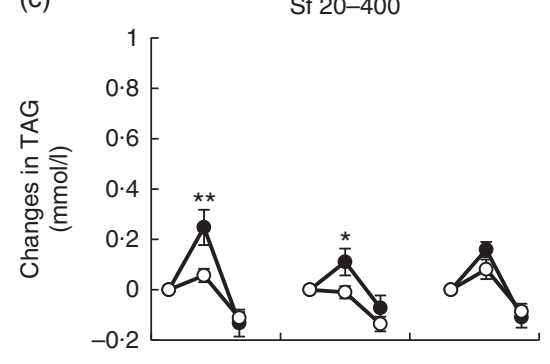

(e)

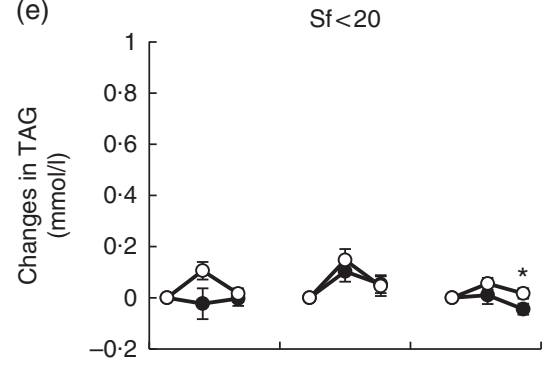

(g)

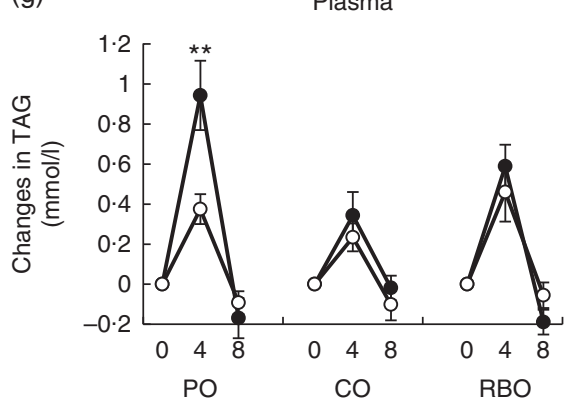

(b) Sf>400

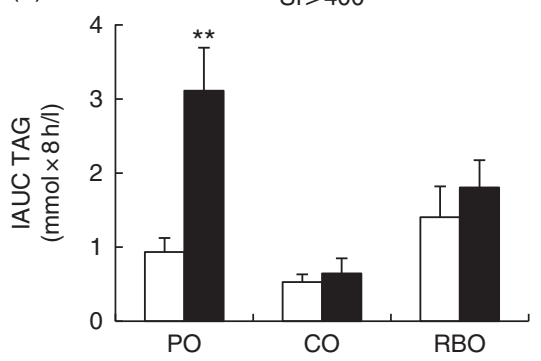

(d)

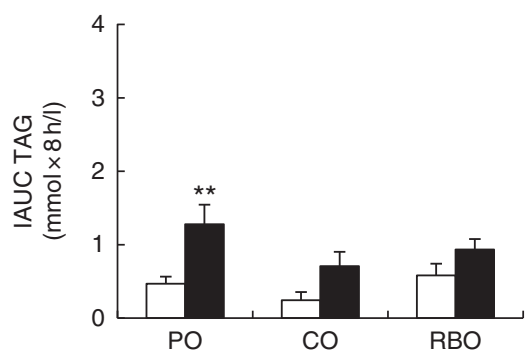

(f)

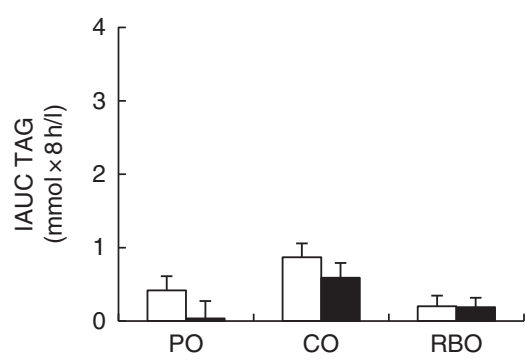

(h)

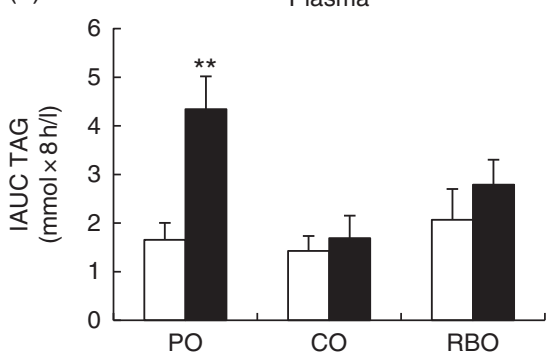

Fig. 2. Changes in TAG concentration ((a), (c), (e), (g)) and incremental AUC (IAUC) of TAG ((b), (d), (f), (h)) in Svedberg flotation rate (Sf) $>400$, Sf $20-400$, Sf $<20$ fraction and plasma following mixed meal containing palm oil (PO), coconut oil (CO) and rice bran oil (RBO). represents hyper-responder (HR) group; $O$ represents normo-responder (NR) group. ${ }^{\star} P$ value $<0.05,{ }^{* *} P$ value $<0.01$, against NR group.

generally strongly associated with the apo B-48 IAUC for the Sf $>400$ and the Sf $20-400$ fractions in HR subjects across all dietary treatment arms. However, measures of fat-free tissue, or conversely adiposity, were not associated with the $\mathrm{Sf}<20$ remnant fraction in HR subjects. In the HR subjects, insulin sensitivity indicated by HOMA-IR score and fasting plasma insulin concentration were strongly associated with the apo B48 IAUC in the Sf $>400$ and to a lesser extent the Sf 20-400 fraction in subjects given RBO or CO. However, the association was not evident in HR subjects when challenged with the PO-enriched meal. Fasting TAG, often considered a surrogate marker of chylomicronaemia, was not associated with chylomicron abundance in any of the lipoprotein fractions or dietary treatment arms considered in HR subjects. Instead, strong associations between fasting apo B-48 concentration and apo B-48 IAUC were observed across all dietary treatment arms in the HR group.

In NR subjects, fat-free mass did not correlate with apo B-48 IAUC for any of the lipoprotein fractions against any of the three meal challenges provided, contrasting with the findings in HR subjects. HOMA and fasting plasma insulin showed association 
Table 3. Correlations between apo B-48 incremental AUC (IAUC) (0-8h) in different fractions following palm oil (PO), coconut oil (CO) and rice bran oil (RBO) meal and baseline metabolic parameters in normo-responder (NR) $(n 16)$ and hyper-responder (HR) $(n 10)$ groups

\begin{tabular}{|c|c|c|c|c|c|c|c|c|c|c|c|c|}
\hline \multirow{2}{*}{$\begin{array}{l}R \text { between apo B-48 } \\
\text { IAUC and parameters }\end{array}$} & \multicolumn{4}{|c|}{$\mathrm{PO}$} & \multicolumn{4}{|c|}{$\mathrm{CO}$} & \multicolumn{4}{|c|}{ RBO } \\
\hline & Plasma & $\mathrm{Sf}>400$ & Sf $20-400$ & $\mathrm{Sf}<20$ & Plasma & $\mathrm{Sf}>400$ & Sf $20-400$ & $\mathrm{Sf}<20$ & Plasma & $\mathrm{Sf}>400$ & Sf $20-400$ & Sf $<20$ \\
\hline \multicolumn{13}{|l|}{ NR } \\
\hline Apo B-48 & -0.211 & -0.629 & -0.132 & -0.193 & -0.246 & -0.411 & -0.343 & -0.046 & 0.053 & -0.071 & -0.115 & 0.191 \\
\hline TAG & 0.2 & -0.211 & 0.154 & -0.029 & -0.075 & 0.139 & 0.171 & -0.2 & $0.715^{\star \star}$ & 0.456 & 0.429 & $0.635^{\star *}$ \\
\hline Total cholesterol & 0.35 & -0.046 & 0.221 & 0.086 & -0.082 & 0.036 & 0.086 & -0.161 & 0.471 & 0.3 & 0.476 & 0.321 \\
\hline LDL-cholesterol & $0.611^{\star}$ & 0.054 & 0.468 & 0.389 & 0.179 & 0.304 & 0.354 & 0.057 & $0.5^{\star}$ & 0.279 & 0.479 & 0.406 \\
\hline HDL-cholesterol & -0.47 & 0.032 & -0.484 & -0.509 & -0.377 & -0.495 & -0.399 & -0.273 & -0.205 & -0.115 & -0.13 & -0.219 \\
\hline Non-HDL-cholesterol & $0.525^{\star}$ & -0.054 & 0.386 & 0.329 & 0.122 & 0.227 & 0.273 & 0.013 & $0.527^{*}$ & 0.277 & 0.446 & 0.455 \\
\hline Glucose & -0.009 & -0.307 & -0.036 & 0.172 & -0.057 & 0.084 & 0.025 & 0.001 & 0.001 & 0.087 & 0.041 & -0.015 \\
\hline Insulin & -0.107 & $0 \cdot 121$ & 0.089 & -0.439 & -0.182 & 0.136 & 0.096 & -0.425 & 0.432 & $0.553^{*}$ & $0.600^{*}$ & 0.059 \\
\hline HOMA-IR & -0.104 & 0.125 & 0.096 & -0.429 & -0.171 & 0.154 & 0.114 & -0.429 & 0.421 & $0.559^{\star}$ & $0.606^{*}$ & 0.026 \\
\hline $\mathrm{BMI}$ & 0.3 & -0.132 & 0.404 & 0.279 & 0.189 & 0.143 & 0.279 & 0.118 & 0.385 & -0.091 & 0.079 & $0.612^{*}$ \\
\hline Waist circumference & 0.418 & 0.068 & $0.575^{*}$ & 0.157 & 0.232 & 0.382 & 0.479 & 0.036 & $0.626^{\star *}$ & 0.226 & 0.432 & $0.662^{\star \star}$ \\
\hline Fat-free mass index & -0.097 & -0.311 & 0.184 & 0.154 & 0.157 & 0.197 & 0.175 & 0.055 & -0.087 & -0.093 & -0.138 & -0.105 \\
\hline Systolic & -0.011 & -0.095 & 0.018 & 0.004 & 0.009 & 0.263 & 0.309 & -0.075 & 0.187 & 0.175 & 0.108 & 0.196 \\
\hline Diastolic & 0.141 & 0.032 & 0.404 & -0.123 & 0.079 & 0.481 & 0.436 & -0.166 & 0.394 & 0.202 & 0.252 & 0.284 \\
\hline \multicolumn{13}{|l|}{ HR } \\
\hline Apo B-48 & $0.810^{\star}$ & 0.643 & $0.952^{\star \star}$ & 0.667 & $0 \cdot 806^{\star \star}$ & 0.588 & $0.721^{\star}$ & 0.345 & 0.588 & 0.43 & $0.758^{*}$ & 0.261 \\
\hline TAG & 0.357 & 0.452 & 0.5 & -0.024 & 0.358 & 0.539 & 0.564 & -0.224 & 0.37 & 0.418 & 0.479 & -0.079 \\
\hline Total cholesterol & 0.263 & 0.001 & 0.419 & 0.168 & -0.085 & -0.146 & 0.043 & -0.122 & -0.079 & 0.067 & 0.267 & -0.328 \\
\hline LDL-cholesterol & 0.214 & -0.167 & 0.357 & 0.238 & 0.006 & -0.152 & 0.042 & 0.079 & -0.03 & -0.018 & 0.248 & -0.2 \\
\hline HDL-cholesterol & 0.263 & -0.228 & 0.12 & 0.311 & -0.354 & -0.591 & -0.433 & -0.03 & -0.439 & -0.396 & -0.165 & -0.299 \\
\hline Non-HDL-cholesterol & 0.204 & 0.001 & 0.395 & 0.072 & 0.073 & 0.061 & 0.213 & -0.073 & 0.122 & 0.182 & 0.359 & -0.195 \\
\hline Glucose & 0.216 & 0.335 & 0.539 & -0.084 & 0.31 & 0.529 & 0.547 & -0.146 & 0.322 & $0.675^{\star}$ & $0.632^{*}$ & -0.255 \\
\hline Insulin & -0.048 & 0.476 & 0.024 & -0.357 & 0.321 & $0.770^{\star \star}$ & 0.6 & -0.2 & 0.176 & $0.721^{*}$ & 0.358 & -0.261 \\
\hline HOMA-IR & -0.073 & 0.512 & 0.049 & -0.439 & 0.264 & $0.742^{*}$ & 0.62 & -0.313 & $0 \cdot 117$ & $0.706^{*}$ & 0.325 & -0.325 \\
\hline $\mathrm{BMI}$ & -0.405 & -0.619 & -0.595 & -0.333 & -0.042 & 0.018 & $-0 \cdot 164$ & 0.333 & 0.079 & -0.176 & -0.273 & 0.188 \\
\hline Waist circumference & 0.071 & -0.405 & -0.143 & 0.19 & 0.2 & 0.03 & -0.115 & 0.612 & 0.285 & -0.152 & 0.018 & 0.333 \\
\hline Fat-free mass index & 0.635 & $0.731^{*}$ & 0.659 & 0.407 & $0.894^{\star \star}$ & $0.833^{\star \star}$ & $0.796^{\star \star}$ & 0.353 & $0.766^{\star \star}$ & 0.48 & $0.632^{*}$ & 0.498 \\
\hline Systolic & -0.095 & -0.19 & -0.095 & -0.262 & 0.152 & 0.333 & 0.188 & 0.164 & 0.115 & 0.309 & 0.248 & -0.224 \\
\hline Diastolic & -0.429 & -0.452 & -0.571 & -0.357 & -0.164 & -0.055 & -0.115 & 0.188 & -0.382 & -0.079 & -0.212 & -0.358 \\
\hline
\end{tabular}

${ }^{\star} P<0.05,{ }^{* \star} P<0.01$ (Spearman's correlation).

in the Sf $>400$ and the Sf $20-400$ for the RBO challenge, but this was not indicated for either the PO or CO meal challenges. In NR group, apo B-48 IAUC in $\mathrm{Sf}<20$ was also strongly associated with waist circumference and BMI following RBO meal.

For HR and NR subjects, there was no significant association between total cholesterol, LDL-cholesterol, HDL-cholesterol, fasting glucose or blood pressure and IAUC apo B-48 responses with the lipoprotein fractions indicated.

\section{Discussion}

This study explored the postprandial plasma distribution of apo $\mathrm{B}-48$ in normolipidaemic subjects following ingestion of isoenergetic meals enriched in either $\mathrm{PO}, \mathrm{RBO}$ or CO. Approximately $40 \%$ of subjects studied demonstrated substantially exaggerated postprandial lipaemia in response to the SFA-rich PO diet (HR group), despite that these subjects did not exhibit an increased fasting concentration of chylomicron particles compared with the NR group. Moreover, significant heterogeneity was found within the responses of the otherwise healthy subjects with normal fasting plasma lipid concentrations, implying variation is due to the individual determinants of chylomicron homoeostasis.

The rationale to consider HR $v$. NR was driven based on the findings from Sato et al. ${ }^{(29)}$. Their results suggest that normolipidaemic subjects with TAG concentration postprandial $\geq 1.7 \mathrm{mmol} / \mathrm{l}$ may have a delayed clearance of TRL. The finding of the present study demonstrates that consideration of heterogeneity based on classical metabolic parameters such as the metabolic syndrome did not effectively segregate those with the exaggerated response to the SFA-rich meal. Hence analogous to classical dietary challenge assessment, we instead considered the postprandial response and set a cut-off value of $1.7 \mathrm{mmol} / \mathrm{l} \mathrm{TAG}$ at peak postprandial phase based on recommendation below this concentration for fasting ${ }^{(36)}$.

The study confirms that contrary to historic considerations, the majority of fasting chylomicrons are within the potentially pro-atherogenic Sf $<20$ fraction $(70-75 \%)^{(37)}$. In both HR and NR subjects the most substantive apo B-48 IAUC response was realised in the $\mathrm{Sf}<20$ fraction, at least twice that compared with the Sf $20-400$ or the Sf $>400$ fractions. The findings indicate that following the ingestion of dietary fats, chylomicronaemia is principally realised in pro-atherogenic particles, which can be delivered through transcytotic processes directly to the subendothelial space. This study demonstrates that within normolipidaemic subjects significant heterogeneity in the magnitude and duration of hyper-remnantaemia is dependent both on the nature of the fatty acids ingested and the likely individuals' metabolic response. A significant subset of individuals studied had substantially elevated apo B-48 IAUC, specifically indicated when consuming a fat challenge rich in SFA. Paradoxically, the same HR subjects had comparable apo B-48 response and distribution between lipoprotein fractions to NR subjects when challenged with meals containing either RBO or CO. The metabolic aberration in these normolipidaemic HR subjects in response to a PO-enriched fat challenge was not detected in correlation analysis by indices of the metabolic syndrome. 
The study findings reiterate that fasting plasma TAG is a poor indicator of chylomicronaemia and potential postprandial atherogenicity $^{(38)}$. It emphasises the merits of considering specifically, chylomicron remnant homoeostasis and response in the context of atherogenic risk. Few studies address the latter, despite the majority of life being spent in the postprandial and absorptive state.

Apo B-48, an equivocal marker of chylomicron homoeostasis and distribution, was principally indicated within the smallest and most dense plasma lipoprotein fraction measured $(\mathrm{Sf}<20)$. These particles typically have a diameter $30-55 \mathrm{~nm}$, sufficient to be captured within the non-specific transcytotic vesicles that deliver small constant amounts of plasma lipoprotein to the subendothelial space $(<70 \mathrm{~nm})^{(16,20)}$. It is a reasonable proposition therefore that a greater understanding of chylomicron atherogenicity would require better appreciation of distributional considerations in the absorptive state; a pathophysiological period that occupies the majority of our 24-h-d. In NR subjects, the plasma response and the distribution of apo B-48 was comparable for dietary challenges enriched in either PO, $\mathrm{RBO}$ or CO suggesting that vascular exposure was otherwise equivalent. In this context, atherogenic risk might be similar. However, other critical factors in the atherogenic cascade including entrapment, uptake by macrophages, mitochondrial respiratory activity or stimulation of inflammatory pathways may still render chylomicron remnants derived from $\mathrm{PO}$ a greater risk than remnants derived from $\mathrm{RBO}$ or $\mathrm{CO}$ in $\mathrm{NR}$ subjects.

In normotriglyceridaemic HR individuals, atherogenic risk may be exacerbated beyond the effects of the fatty acid per se, but as a consequence of heightened vascular exposure to chylomicrons. Despite the metabolic aberration in these individuals principally being indicated in the lipolytic cascade, nonetheless the substantive accumulation and response was for the large part realised within the dense $\mathrm{Sf}<20$ fraction. On the basis that chylomicronaemia was observed in HR subjects following the PO meal and not when challenged with either RBO or $\mathrm{CO}$, we suggest that the metabolic defect is indicated specifically in the metabolism of the particles enriched in PO. Given that the response to PO was not exaggerated in all subjects (only in the HR group), one interpretation is that the defect may not be due to the fatty acid composition in de novo chylomicrons. Instead, the defects may be due to the complement of regulating apo that are associated with chylomicrons, which differs between individuals (between NR and HR groups). Differential expression of key apo involved in lipolysis (e.g. apo CII/CIII), or remnant clearance (apo E isoforms) may be a genetic point of synergistic regulation of PO-enriched chylomicrons $^{(39,40)}$. Meal rich in SFA (PO-based meal) has been reported to modulate the amount of apoC-II, apoC-III and apo E than meal rich in MUFA and PUFA ${ }^{(40)}$. Since the exaggerated apo $\mathrm{B}-48$ response was observed in the HR group following $\mathrm{PO}$ meal but not CO or RBO meal or in the NR group, this raises the possibility of interactions between type of dietary fatty acid (PO-based meal) and individual metabolic response. Alternatively, abundance or isoforms of lipolytic enzymes or receptor clearance proteins may explain the interactive effects with dietary lipids on chylomicron abundance. The latter seems less likely given that there was no difference between NR and $\mathrm{HR}$ in response to RBO- or CO-enriched meal challenges. Correlation analysis indicated for both NR and HR subjects, a consistently strong association between fat-free mass and apo B-48 homoeostasis, particularly within the Sf $>400$ and the Sf 20-400 fractions. In the absence of a frank lipolytic defect in HR subjects (normal fasting TAG, normal response to RBO and $\mathrm{CO})$, one explanation for the heightened apo B-48 response in HR subjects principally within Sf $>400$ and Sf 20-400 (but not in $\mathrm{Sf}<20$ ) might be in a modest hydrolytic defect in muscle tissue-associated LPL, given the consistent association between fat-free mass (inverse association with fat mass) and apo B-48 abundance within larger more lipoprotein fractions. The latter is an interesting consideration given that HR subjects had greater fat-free mass and that muscle and adipose tissue LPL are differentially regulated ${ }^{(41)}$. Insulin stimulates adipose tissue LPL but inhibits expression of muscle tissue LPL. Meals high in SFA content have been reported to decrease insulin sensitivity ${ }^{(42,43)}$ via accumulation of TAG and ceramide in muscle, activation of protein kinase $\mathrm{C}, \mathrm{NF}-\kappa \mathrm{B}$ and the subsequent inflammatory genes $^{(44)}$. By extension, muscle-mediated LPL activity may have been attenuated in HR subjects ${ }^{(45)}$. Nonetheless, the data from this study suggest that any such putative tissue-bed lipolytic defect in HR subjects would be a differential interaction between enzyme and substrate (chylomicron), because RBO and $\mathrm{CO}$ challenges were comparable between HR and NR subjects $^{(11)}$.

Measures of obesity suggested the HR subjects exhibited greater central obesity and were more overweight than the NR subjects, however there was no evidence of insulin resistance, or the metabolic syndrome based on current combined criteria per se. Insulin sensitivity is indicated in the context of glucose metabolism and plasma insulin homoeostasis, surrogate markers that may be inappropriate for identifying key regulatory determinants of chylomicron homoeostasis and metabolism.

In these otherwise healthy $\mathrm{HR}$, the findings indicate that classical markers of plasma lipid homoeostasis provide no valuable insight into chylomicron metabolism, remnant homoeostasis and by extension atherogenic risk. Plasma TAG, cholesterol within the LDL, HDL and non-HDL-cholesterol were otherwise normal and not associated with apo B-48 IAUC measures.

The limitations of the present study are the relatively small number of participants and the unbalanced sex distribution between groups, nonetheless the study was sufficiently powered to demonstrate treatment effects. In the present study, the limited number of postprandial time points for blood collection in each postprandial course may miss an early or late apo B-48 or TAG peak resulting in underestimation or overestimation of apo B-48 postprandial response. However three time points blood collection for oral fat tolerance test have been shown to have high correlation with hourly blood collections following an oral fat tolerance test ${ }^{(46)}$. The current guidelines adopted by several countries have used non-fasting TAG concentration of 1.89 or $2 \mathrm{mmol} / \mathrm{l}$ or more as the cut-off value for increased risk of CVD events ${ }^{(47)}$. Further examination with a higher cut-off value (i.e. $2 \mathrm{mmol} / \mathrm{l}$ ) is warranted. Analysis of apo C, apo $\mathrm{E}$ and LPL are necessary due to their role in lipolysis and remnant 
uptake. In the present study, we did not examine LPL activity and those apo (apo C-II, apo C-III, apo E) therefore we could not determine which key apo or enzymes interacts synergistically with dietary fatty acid in the HR group.

In conclusion, we have demonstrated that some healthy normotriglyceridaemic individuals are susceptible to develop exaggerated chylomicron remnantaemia in response to meals enriched in SFA derived from PO. HR subjects were susceptible to a greater exposure of atherogenic small-sized remnants in response to PO-enriched meals with modest or no effects indicated for $\mathrm{RBO}$ and $\mathrm{CO}$. Silent hyper-remnantaemia in response to $\mathrm{PO}$-enriched meals in some normolipidemic subjects may contribute to atherogenic risk, but not in others.

\section{Acknowledgements}

The authors gratefully acknowledge the participants, Kerrie Collier and Arielle Hyland for their help in participant recruitments, and Edwin Junaldi for the assistance in GC. J. C. L. M. is supported by the Australian National Health and Medical Research Council. D. I. is a recipient of Australian Award Scholarship.

The work reported in this manuscript was supported by D. I.'s PhD candidacy funds provided by Curtin University. The project was in part supported by an Australian National Health and Medical Research Council Project Grant.

Conceived and designed the experiments: D. I., J. C. L. M., K. M. S.-C., M. J. S. and A. P. J. Performed the experiments: D. I. and A. P. J. Analysed the data: D. I. assisted by A. P. J. and J. C. L. M. Wrote the manuscript: D. I. and J. C. L. M. Reviewed and critically revised the manuscript for important intellectual content: J. C. L. M., K. M. S.-C, M. J. S. and A. P. J. All authors approved the final version of the manuscript.

The authors declare that there are no conflicts of interest.

\section{References}

1. Williams CM (1997) Postprandial lipid metabolism: effects of dietary fatty acids. Proc Nutr Soc 56, 679-692.

2. Dallinga-Thie GM, Franssen R, Mooij HL, et al. (2010) The metabolism of triglyceride-rich lipoproteins revisited: new players, new insight. Atherosclerosis 211, 1-8.

3. Cooper AD (1997) Hepatic uptake of chylomicron remnants. I Lipid Res 38, 2173-2192.

4. Karpe F, Olivecrona T, Hamsten A, et al. (1997) Chylomicron/ chylomicron remnant turnover in humans: evidence for margination of chylomicrons and poor conversion of larger to smaller chylomicron remnants. J Lipid Res 38, 949-961.

5. Redgrave TG (2004) Chylomicron metabolism. BiochemSoc Trans 32, 79-82.

6. Mamo JCL, Proctor SD \& Smith D (1998) Retention of chylomicron remnants by arterial tissue; importance of an efficient clearance mechanism from plasma. Atherosclerosis 141, Suppl. 1, S63-S69.

7. Alipour A, Valdivielso P, Elte JWF, et al. (2010) ApoB48 as a marker of atherosclerosis in patients with familial lipid disorders, type 2 diabetes mellitus and coronary artery disease. Atheroscler Suppl 11, 68-69.

8. Sakai N, Uchida Y, Ohashi K, et al. (2003) Measurement of fasting serum apoB-48 levels in normolipidemic and hyperlipidemic subjects by ELISA. J Lipid Res 44, 1256-1262.
9. Karpe F, Steiner G, Uffelman K, et al. (1994) Postprandial lipoproteins and progression of coronary atherosclerosis. Atherosclerosis 106, 83-97.

10. Su JW, Nzekwu MMU, Cabezas MC, et al. (2009) Methods to assess impaired post-prandial metabolism and the impact for early detection of cardiovascular disease risk. Eur J Clin Invest 39, $741-754$

11. Adiels M, Matikainen N, Westerbacka J, et al. (2012) Postprandial accumulation of chylomicrons and chylomicron remnants is determined by the clearance capacity. Atherosclerosis 222, 222-228.

12. Kinoshita M, Ohnishi H, Maeda $\mathrm{T}$, et al. (2009) Increased serum apolipoprotein B48 concentration in patients with metabolic syndrome. $J$ Atheroscler Thromb 16, 517-522.

13. Dane-Stewart CA, Watts GF, Barrett PHR, et al. (2003) Chylomicron remnant metabolism studied with a new breath test in postmenopausal women with and without type 2 diabetes mellitus. Clin Endocrinol (Oxf) 58, 415-420.

14. Dane-Stewart CA, Watts GF, Mamo JCL, et al. (2001) Elevated apolipoprotein B-48 and remnant-like particle-cholesterol in heterozygous familial hypercholesterolaemia. Eur J Clin Invest 31, 113-117.

15. Karpe F, Hellénius ML \& Hamsten A (1999) Differences in postprandial concentrations of very-low-density lipoprotein and chylomicron remnants between normotriglyceridemic and hypertriglyceridemic men with and without coronary heart disease. Metabolism 48, 301-307.

16. Simionescu M \& Simionescu N (1991) Endothelial transport of macromolecules: transcytosis and endocytosis. A look from cell biology. Cell Biol Rev 25, 1-78.

17. Dallinga-Thie GM, Kroon J, Borén J, et al. (2016) Triglyceriderich lipoproteins and remnants: targets for therapy? Curr Cardiol Rep 18, 67

18. Proctor SD \& Mamo JCL (1998) Retention of fluorescentlabelled chylomicron remnants within the intima of the arterial wall: evidence that plaque cholesterol may be derived from post-prandial lipoproteins. Eur J Clin Invest 28, 497-503.

19. Fielding CJ (1992) Lipoprotein receptors, plasma cholesterol metabolism, and the regulation of cellular free cholesterol concentration. FASEB J 6, 3162-3168.

20. Proctor SD, Vine DF \& Mamo JCL (2004) Arterial permeability and efflux of apolipoprotein B-containing lipoproteins assessed by in situ perfusion and three-dimensional quantitative confocal microscopy. Arterioscler Thromb Vasc Biol 24, 2162-2167.

21. Irawati D, Mamo JCL, Soares MJ, et al. (2015) Hypertriglyceridemic subjects exhibit an accumulation of small dense chylomicron particles in the fasting state. Atherosclerosis 243, 236-241.

22. Campos H, Khoo C \& Sacks FM (2005) Diurnal and acute patterns of postprandial apolipoprotein B-48 in VLDL, IDL, and LDL from normolipidemic humans. Atherosclerosis 181, 345-351.

23. Williams CM (1998) Dietary interventions affecting chylomicron and chylomicron remnant clearance. Atherosclerosis $\mathbf{1 4 1}$, Suppl. 1, S87-S92.

24. Williams CM, Bateman PA, Jackson KG, et al. (2004) Dietary fatty acids and chylomicron synthesis and secretion. Biochem Soc Trans 32, 55-58.

25. Lamarche B \& Couture P (2015) Dietary fatty acids, dietary patterns, and lipoprotein metabolism. Curr Opin Lipidol 26, $42-47$.

26. Mensink RP, Zock PL, Kester ADM, et al. (2003) Effects of dietary fatty acids and carbohydrates on the ratio of serum total to HDL cholesterol and on serum lipids and 
apolipoproteins: a meta-analysis of 60 controlled trials. $\mathrm{Am} \mathrm{J}$ Clin Nutr 77, 1146-1155.

27. Esser D, van Dijk SJ, Oosterink E, et al. (2013) A high-fat SFA, MUFA, or $n$-3 PUFA challenge affects the vascular response and initiates an activated state of cellular adherence in lean and obese middle-aged men. $J$ Nutr 143, 843-851.

28. Ruiz-Núñez B, Dijck-Brouwer DAJ \& Muskiet FAJ (2016) The relation of saturated fatty acids with low-grade inflammation and cardiovascular disease. J Nutr Biochem 36, 1-20.

29. Sato I, Ishikawa Y, Ishimoto A, et al. (2009) Significance of measuring serum concentrations of remnant lipoproteins and apolipoprotein B-48 in fasting period. $J$ Atheroscler Thromb 16, 12-20.

30. Havel RJ, Eder HA \& Bragdon JH (1955) The distribution and chemical composition of ultracentrifugally separated lipoproteins in human serum. J Clin Invest 34, 1345-1353.

31. James AP \& Mamo JC (2012) Consumption of low doses of fat prevents the postprandial rise in chylomicron particle concentration and remnant accumulation in healthy normolipidaemic males. J Nutr Sci 1, 1-8.

32. Bairaktari E, Hatzidimou K, Tzallas C, et al. (2000) Estimation of LDL cholesterol based on the Friedewald formula and on apo B levels. Clin Biochem 33, 549-555.

33. Matthews DR, Hosker JP \& Rudenski AS (1985) Homeostasis model assessment: insulin resistance and $\beta$-cell function from fasting plasma glucose and insulin concentrations in man. Diabetologia 28, 412-419.

34. Kinoshita M, Kojima M, Matsushima T, et al. (2005) Determination of apolipoprotein B-48 in serum by a sandwich ELISA. Clin Chim Acta 351, 115-120.

35. Matthews DR (1988) Time series analysis in endocrinology. Acta Paediatr Scand, Suppl 77, 55-62.

36. Miller M, Stone NJ, Ballantyne C, et al. (2011) Triglycerides and cardiovascular disease: a scientific statement from the American Heart Association. Circulation 123, 2292-2333.

37. Karpe F, Bell M, Bjorkegren J, et al. (1995) Quantification of postprandial triglyceride-rich lipoproteins in healthy men by retinyl ester labeling and simultaneous measurement of apolipoproteins B-48 and B-100. Arterioscler Thromb Vasc Biol 15, 199-207.
38. Irawati D, Mamo JCL, Dhaliwal SS, et al. (2016) Plasma triglyceride and high density lipoprotein cholesterol are poor surrogate markers of pro-atherogenic chylomicron remnant homeostasis in subjects with the metabolic syndrome. Lipids Health Dis 15, 1-9.

39. Schwarzova L, Hubacek JA \& Vrablik M (2016) Genetic predisposition of human plasma triglyceride concentrations. Physiol Res 64, S341-S354.

40. Jackson KG, Wolstencroft EJ, Bateman PA, et al. (2005) Greater enrichment of triacylglycerol-rich lipoproteins with apolipoproteins E and C-III after meals rich in saturated fatty acids than after meals rich in unsaturated fatty acids. Am J Clin Nutr 81, 25-34.

41. Mead JR, Irvine SA \& Ramji DP (2002) Lipoprotein lipase: structure, function, regulation, and role in disease. $J$ Mol Med 80, 753-769.

42. Galgani JE, Uauy RD, Aguirre CA, et al. (2008) Effect of the dietary fat quality on insulin sensitivity. Br J Nutr 100, 471-479.

43. Riccardi G, Giacco R \& Rivellese AA (2004) Dietary fat, insulin sensitivity and the metabolic syndrome. Clin Nutr $\mathbf{2 3}$, 447-456.

44. Kennedy A, Martinez K, Chuang CC, et al. (2009) Saturated fatty acid-mediated inflammation and insulin resistance in adipose tissue: mechanisms of action and implications. J Nutr 139, $1-4$.

45. Annuzzi G, Giacco R, Patti L, et al. (2008) Postprandial chylomicrons and adipose tissue lipoprotein lipase are altered in type 2 diabetes independently of obesity and whole-body insulin resistance. Nutr Metab Cardiovasc Dis 18, 531-538.

46. Maraki M, Aggelopoulou N, Christodoulou N, et al. (2011) Validity of abbreviated oral fat tolerance tests for assessing postprandial lipemia. Clin Nutr 30, 852-857.

47. Nordestgaard BG, Langsted A, Mora S, et al. (2016) Fasting is not routinely required for determination of a lipid profile: clinical and laboratory implications including flagging at desirable concentration cut-points - a joint consensus statement from the European Atherosclerosis Society and European Federation of Clinical Chemistry and Laboratory Medicine. Eur Heart J 37, 1944-1958. 